\title{
Indikatoren für Verstehen, Missverstehen und Nichtverstehen in Gesprächen
}

\author{
Magdalena Putz (Bozen)
}

\begin{abstract}
This paper discusses understanding, misunderstanding and not-understanding of German dialect expressions. Reactions indicating understanding, misunderstanding and notunderstanding in discourse are listed and examples from a medical corpus, analysing the reactions of non-dialect speakers talking to dialect speakers, are discussed.
\end{abstract}

\section{$1 \quad$ Hintergrund}

Den Hintergrund der Auseinandersetzung mit Verstehen, Missverstehen und Nichtverstehen bildet das Dissertationsprojekt der Verfasserin, innerhalb dessen verständnishemmende Einheiten des Südtiroler Dialekts ${ }^{1}$ identifiziert werden sollen. Ziel des Projekts ist die Ausarbeitung von Referenzmaterialien für nicht Dialekt sprechende in Südtirol praktizierende Basisärzte/innen italienischer Muttersprache, die in Visitengesprächen Schwierigkeiten mit dem Verstehen ihrer Dialekt sprechenden Patient/innen haben. Zu diesem Zweck werden Visitengespräche aufgenommen, transkribiert und auf für das Verständnis problematische Einheiten untersucht.

Der erste Teil dieses Beitrags geht auf Verstehen, Missverstehen und Nichtverstehen in Gesprächen ein. Der zweite Teil befasst sich mit verbalen und nonverbalen Einheiten, welche Verständnis, Missverstehen und Nichtverstehen in Gesprächen signalisieren. Die Verfasserin geht davon aus, dass ein/e Außenstehende/ $\mathrm{r}^{2}$ anhand von bestimmten Elementen im Gespräch erkennen kann, wo verstanden wird, wo Missverständnisse auftreten und wo Nichtverstehen gegeben ist. Anhand der identifizierten Indizien sollen Rückschlüsse auf verstehenshemmende Einheiten gezogen werden, die in das geplante Referenzmaterial einfließen sollen. Eine derartige Herangehensweise an verstehenshemmende sprachliche Einheiten wird im letzten Teil exemplarisch anhand von Beispielen aus dem Visitenkorpus dargestellt.

\section{Verstehen im Gespräch}

Die Untersuchung von Verstehen beschäftigt mehrere Disziplinen, wie die Sozial- und Bewusstseinstheorie, die Hermeneutik und die Kognitionspsychologie (cf. Deppermann im Druck). Entsprechend der verschiedenen Herangehensweisen sind auch die Definitionen des Verstehensbegriffs vielfältig. Bei den teilweise konkurrierenden Theorien der einzelnen Disziplinen zum Verstehensbegriff ist noch unklar, wie diese Theorien miteinander in Einklang gebracht werden können (cf. Schäflein-Armbruster 1994: 495).

\footnotetext{
${ }^{1}$ Der Begriff "Dialekt" wird hier vereinfacht verwendet und wird der Existenz der verschiedenen Dialekte und Varietäten des Deutschen auf dem Dialekt-Hochsprache-Kontinuum in Südtirol nicht gerecht.

2 Zur Perspektive von Missverständnissen cf. Hinnenkamp (1998: 205).
} 
Für die Beurteilung von "Verstehen" sind mehrere Faktoren relevant: Verschiedene Hörer/innen stellen unterschiedliche Ansprüche an ihr Verständnis, jede/r Hörer/in versteht bei ein und demselben Gehörten unterschiedlich viel, aber auch unterschiedliche Inhalte (cf. Schäflein-Armbruster 1994: 494). Das Verstehen von Gehörtem betrifft außerdem mehrere Elemente eines Gesprächs: akustisches Verstehen, Verstehen der Sprecher- und Hörerrolle, Verstehen der Wortbedeutung, der Satzbedeutung, der Äußerungsbedeutung, des Gesprächsthemas und der thematischen Handlung, des Rahmens und der Textart, der Gesprächsmaximen und des Textbezugs (cf. Bublitz 2001: 1332-1335).

Unabhängig von den jeweiligen Blickwinkeln der Disziplinen, die sich mit dem Verstehen auseinandersetzen - sowie die eben genannten Faktoren und Elemente übergreifend - ist die Tatsache, dass Verstehen dem Handeln untergeordnet ist, d. h. Verstehen hat keinen Selbstzweck, sondern wird "nur soweit verdeutlicht und bearbeitet, wie es Belange der Handlungssteuerung, -koordination und -progression erfordern. Verstehen ist konstitutiv für Handeln, diesem aber untergeordnet" (Deppermann im Druck). Rehbein drückt dies folgendermaßen aus: "Verständigung richtet sich danach, was mit der anstehenden Kommunikation faktisch erreicht werden soll." (Rehbein 1985: 11, zitiert nach Rost-Roth 1994: 29).

Weiters von den einzelnen Disziplinen unabhängig scheint die Messbarkeit von Verstehen anhand eines Kontinuums zu sein, wobei jedoch Verstehen auf diesem Kontinuum schwer festzumachen ist. Hundertprozentiges Verstehen ist sehr unwahrscheinlich und empirisch kaum beweisbar (cf. Falkner 2007: 196f.). Entsprechend seiner pragmatischen Einstellung zum Verstehen meint Deppermann (im Druck), es gehe auf diesem Kontinuum oft darum, ob der/die Gesprächspartner/in die Aussage als hinreichend verständlich klassifiziert oder nicht. Auf der anderen Seite des Kontinuums als Gegenpol einer erfolgreichen Kommunikation siedelt Rost-Roth (1994: 36) sowohl das Missverstehen als auch das Nichtverstehen an.

Der Übergang von Verstehen zu Missverstehen und Nichtverstehen kann aufgrund der eingangs genannten Faktoren keine klare Grenze aufweisen. Als Außenstehende/r wird es außerdem selbst bei noch so großen Detailwissen über die Gesprächsteilnehmer/innen, den Kontext und den übrigen für ein Gespräch wichtige Details unmöglich bleiben, den Verstehensprozess vollständig zu verstehen, d. h. den dokumentierten Verstehensprozess mit Sicherheit an einem Kontinuum festmachen zu können. Abgesehen davon sind Verstehensprozesse selbst den Gesprächsteilnehmer/innen selten bewusst bzw. werden Verstehensprobleme oft nicht als solche erkannt, und könnten deshalb auch von den Gesprächsteilnehmer/innen selbst nicht festgemacht werden.

\subsection{Missverständnis/Missverstehen}

Obwohl Missverstehen auf einen ersten Blick als Gegenteil von gelungener Kommunikation verstanden werden könnte, weist Rost-Roth darauf hin, dass Fehlkommunikation nicht nur ein bedauerliches Resultat von Begegnungen ist, sondern auch gesellschaftlich funktional sein kann (cf. Rost-Roth 1994: 30). Im Folgenden wird jedoch nur auf jene Missverständnisse eingegangen, die vom/von der Sprecher/in nicht intendiert sind.

Die umfangreichste Auseinandersetzung mit Missverständnissen anhand von empirischen Untersuchungen stammt von Hinnenkamp (1998). "Missverständnisse sind ein frequenter und allgegenwärtiger Bestandteil des alltäglichen kommunikativen Daseins ${ }^{3}$." (Hinnenkamp 1998: 314). Die laut Hinnenkamp in der Linguistik am weitesten verbreitete Definition der

\footnotetext{
${ }^{3}$ Zur Allgegenwärtigkeit von Missverständnissen in unterschiedlichen Disziplinen cf. Hinnenkamp 1998: Kapitel 2 "Die Ubiquität von Missverständnissen".
} 
Missverständnisse ist jene der Diskrepanz zwischen Intendiertem und Verstandenem (1998: 55).

Im Gegensatz zum "Verstehen" lassen sich Missverständnisse nach ihrer äußeren und inneren "Manifestanz" ordnen (Hinnenkamp 1998: 117f.). Die äußere Ordnung umfasst manifeste, verdeckte bzw. versteckte und unbemerkte Missverständnisse ${ }^{4}$ : Manifeste Missverständnisse werden von mindestens einem der Sprecher/innen metasprachlich thematisiert. Verdeckte bzw. versteckte Missverständnisse zeigen sich an, werden jedoch nicht also solche angesprochen. Bei der dritten Kategorie handelt es sich um Missverständnisse, die unbemerkt bleiben. Falkner schlägt vor, "das für die Interaktanten manifeste Missverständnis vom durch die Beteiligten unbemerkten Zustand des Missverstehens begrifflich zu unterscheiden". Nach dieser Unterscheidung sei nur das Missverständnis einer systematischen Analyse zugänglich. "Missverstehen dagegen wird ja, solange es unerkannt bleibt, als Verstehen wahrgenommen." (Falkner 2007: 197). ${ }^{5}$

Die Ordnung der Missverständnisse nach inneren Kriterien erfolgt nach dem Typ der Missverständnisse. Äußere und innere Ordnung korrelieren miteinander.

Aus sprechakttheoretischer Sichtweise werden die Elemente, die beim Verstehen bzw. Missverstehen eine Rolle spielen, ebenso in eine äußere und inhaltliche Ebene unterteilt. Die Äußerungsform einer sprachlichen Handlung umfasst hier allerdings die Sprechgeschwindigkeit, die Intonation, die Lautform, die syntaktische Form der Äußerung, die verwendeten lexikalischen Formen u. a. m. (Schäflein-Armbruster 1994: 498). Auf der Inhaltsebene entwirft Falkner eine Typologie anhand derer Missverständnisse auf den Ebenen der Illokution, Proposition und Modifikatoren 6 geortet werden können (cf. Falkner 207: 188).

\subsection{Nichtverstehen}

Analog zum Verstehensbegriff tauchen folgende Begriffe immer weder auf: Missverstehen, Missverständnis, Missverständlichkeit (Hinnenkamp 1998: 123), Verstehensschwierigkeiten (Marti 2001: 29), Verständigungsschwierigkeiten (Rost-Roth 1994), Verstehensprobleme (Schäflein-Armbruster 1994: 493), Verständigungsprobleme (Rost-Roth 1994), fehlschlagende Kommunikation (Rehbein 1985), Fehlkommunikation (Rost-Roth 1994: 10), Problematische Kommunikation (Marti 2001: 14), Unverständlichkeit (Hinnenkamp 1998: 123), kommunikativer Missverfolg (Grimshaw nach Selting 1987: 12). Diese Begriffsvielfalt veranschaulicht die Mannigfaltigkeit und Uneinheitlichkeit der einzelnen Konzepte auf der anderen Seite des Verstehenskontinuums. Für das bezweckte Forschungsvorhaben wird zwischen Missverstehen und Nichtverstehen unterschieden, wobei sich noch die Frage stellen wird, ob eine derartige Unterscheidung tatsächlich sinnvoll ist (u. a. weil eine klare Trennung der beiden Konzepte, wie bereits eingangs erwähnt, unmöglich ist). Hinnenkamp spricht sich für eine Trennung aus: "Unverständlichkeit und Missverständlichkeit werden dialogisch unterschiedlich behandelt und sind konstitutiv für eben diese Unterscheidung." (Hinnenkamp 1998: 123). Prinzipiell wird nämlich Missverstehen solange als Richtig-Verstehen gehandelt, bis es entdeckt wird (Hinnenkamp 1998: 124 oder auch Falkner 1997: 178 zitiert nach Marti 2001: 34).

\footnotetext{
${ }^{4}$ Hinnenkamp unterscheidet zusätzlich zwischen reziprok manifesten und nicht reziprok manifesten Missverständnissen (1998: 315).

${ }^{5} \mathrm{Im}$ vorliegenden Beitrag werden Missverstehen und Missverständnis im Sinne von Hinnenkamp synonym verwendet .

${ }^{6}$ Nach Searle gehören zur inneren Struktur von Handlungen folgende Aspekte: Illokution, Proposition, Referenz, Prädikation (Schäflein Armbruster 1994: 497).
} 
Marti (2001: 33) zweifelt an der Existenz vollkommenen Nichtverstehens. Er geht davon aus, dass bei gegenseitiger Aufmerksamkeit der/die Gesprächspartner/in immer etwas - wenn auch nicht viel - versteht.

Im Zuge der Analysen der zu Grunde liegenden Arbeit erwartet die Verfasserin Fälle von Nichtverstehen aufgrund von unbekannten dialektalen Einheiten. Dass Verstehen im Kontext trotz unbekannter Ausdrücke möglich ist, ist klar. Im Laufe weiterer Analyse soll sich herausstellen, wie wichtig eine Unterscheidung zwischen Missverständnissen und Nichtverstehen zum Zweck der Untersuchung ist.

\section{Indikatoren für Verstehen, Missverstehen und Nichtverstehen im Gesprächs- verlauf}

In diesem Abschnitt werden sprachliche und nonverbale Handlungen, die im Gespräch Verstehen, Missverstehen und Nichtverstehen signalisieren, besprochen. Die Verfasserin geht davon aus, dass man als Außenstehende $/ \mathrm{r}^{7}$ anhand von bestimmten Elementen im Gespräch erkennen kann, wo verstanden wird, wo Missverständnisse auftreten und wo Nichtverstehen gegeben ist.

Verstehen ist ein interaktiver Prozess und wird aktiv von Sprecher/in und Hörer/in angestrebt. (Zu den Strategien der Verständnissicherung von Seiten des Sprechers/der Sprecherin cf. Bublitz 2001.) Verstehen wird im Gespräch von den Gesprächspartner/innen laufend signalisiert und überprüft (cf. Bublitz 2001): Der/die Sprecher/in versucht, durch verschiedene sprachliche Mittel das Verstehen ihrer/seiner Aussage seitens des/der Hörenden zu sichern. Der/die Hörer/in signalisiert seiner-/ihrerseits das Verständnis durch sein/ihr Rückmeldeverhalten $^{8}$. Da der Verstehensakt ein mentaler Prozess ist, kann er im Text nur anhand von Reaktionen bezüglich des Verstehens gemessen werden. In den Reaktionen wird Verstehen, Missverstehen und Nichtverstehen explizit oder implizit geäußert. Explizite Äußerungen sind selbstverständlich leichter zu erkennen als implizite.

\subsection{Verstehen}

Explizite Reaktionen auf Verstehen in Gesprächen werden vielfach durch eine metasprachliche Thematisierung geäußert. In der Regel werden dafür Verben wie verstehen, meinen, wissen verwendet. Verstehen wird jedoch in den meisten Fällen implizit bestätigt und bleibt somit lautlos. Sprachliche Formen, mit denen der/die Hörende Verstehen signalisiert, sind außerdem "Formulierungsaufnahmen, Turnvervollständigungen und -fortführungen, Reformulierungen und Inferenzformulierungen, Verstehensthematisierungen (S.13), Selbstund Fremd-Korrekturen und bestimmte Interjektionen" (cf. Deppermann im Druck), z. B. "Ja, ja, das hab ich schon verstanden."

\subsection{Missverstehen}

Entsprechend der Anordnung von Missverständnissen an einem Kontinuum anhand ihrer Manifestation (cf. Abschnitt 2) können auch die Anzeichen, die auf Missverständnisse hindeuten, skalar angeordnet werden. Die folgende Beschreibung der Signale für Missverständnisse folgt der Anordnung von Hinnenkamp (1998: 117f.):

Explizite metakommunikative Feststellungen von Missverständnissen, die auf der von Hinnenkamp angelegten Skala ganz links angesiedelt sind, dürften nicht schwer zu erkennen sein. Derartige manifeste Missverständnisse werden selbst- oder fremd diagnostiziert, z. B.

\footnotetext{
${ }^{7}$ Zur Perspektive von Missverständnissen siehe Hinnenkamp 1998: 205.

${ }^{8}$ Zur Unmöglichkeit einer strikten Trennung von Sprecher/in und Hörer/in cf. Rath (2001: 1217) und Linz (2007: 43).
} 
"Ich glaube, du hast mich missverstanden" vs. "Ich glaube, ich habe dich missverstanden." Weniger explizit aber immer noch deutlich sind Reaktionen wie "Ach SO war das. Ich dachte...", oder aber "Ach, jetzt verstehe ich!", wo nicht mehr ausgeführt wird, dass ein Missverständnis vorgelegen hat, sondern bei dem nur noch der momentane Verstehensprozess betont wird. Als ein nonverbales relativ deutliches Signal für Missverstehen kann eine verzögerte Reaktionszeit stehen.

In dem von Hinnenkamp untersuchten Korpus werden Hinweise auf Missverständnisse sehr oft mit dem Verb meinen ausgedrückt, z. B. "Ich meinte, dass...", "Ich dachte, du meinst..." (cf. Hinnenkamp 1998: 155-158). Indikativ für Missverständnisse können auch einzelne Wörter wie Interjektionen sein, z. B. ach, ach so (158-162). Missverständnisklärungen werden manchmal auch mit dem Wort nein eingeleitet, z. B. "Nein, wie es DEINer Cousine geht hab ich gefragt".

Weniger explizite Anzeichen finden wir für Missverständnisse, die sich auf dem Manifestationskontinuum in der Mitte befinden. Bei derartigen Missverständnissen werden keine Verben wie meinen, denken usw. verwendet, sondern es wird direkt auf einzelne fragwürdige Elemente eingegangen, z. B. durch eine fragende oder aussagende Wiederholung von missverständlichen Einheiten, z. B. "VerBAUT?"

Je undeutlicher Missverständnisse sich äußern, bzw. je weiter sie sich auf der rechten Seite des Manifestationskontinuums befinden, desto schwieriger wird es, sie an bestimmten Merkmalen zu erkennen: "Klärungssequenzen", "Reparaturen" und "Korrekturen" (Hinnenkamp 1998: 175) können ein Indiz für Missverständnisse sein, z. B. "Ah, ja, in der Schule!". Ebenso kann ein überraschender Tonfall oder ein scheinbar thematischer Widerspruch auf ein Missverständnis hinweisen.

\subsection{Nichtverstehen}

Höchstwahrscheinlich ist es als Außenstehende/r in vielen Fällen nicht möglich zu beurteilen, ob es sich nun um Missverstehen oder um Nichtverstehen handelt. Anhand eines Korpus von Gesprächen zwischen Nicht-Muttersprachler/innen identifiziert Marti Indikatoren, die Nichtverstehen anzeigen und ordnet sie an einem Kontinuum an, innerhalb dessen es - wie auch bei Verstehen und Missverständnissen - explizite und implizite Anzeiger gibt. Die Indikatoren, die Nichtverstehen anzeigen, veranschaulicht Marti in folgender Tabelle (Marti 2001: 51):

\begin{tabular}{|l|l|l|}
\hline \multirow{3}{*}{ Explizite Anzeiger } & \multicolumn{1}{|c|}{ Gruppe } & \multicolumn{1}{c|}{ Kategorie } \\
\hline \multirow{2}{*}{ Implizite Anzeiger } & \multirow{2}{*}{ Ignorieren } & a Signalisation von Nichtverstehen \\
\cline { 3 - 3 } & & b Erfragen von Zusatzinformation \\
\cline { 3 - 3 } & c Wiederholung eines zentralen Elements \\
\cline { 2 - 3 } & Abbrechen & $\begin{array}{l}\text { d Zustimmung trotz teilweisem Nichtverstehen } \\
\text { Missverständnis in Kauf nehmen }\end{array}$ \\
\hline & \begin{tabular}{l} 
f Themawechsel \\
\hline
\end{tabular} & g Lachen \\
\hline & h Abbruch \\
\hline
\end{tabular}

Tabelle 1: Indikatoren von Nichtverstehen (Marti 2001: 51)

Der größte Grad an Explizitheit ist erreicht, wenn das Problem metasprachlich thematisiert wird. Bei dem von Marti untersuchten Korpus werden von Nicht-Muttersprachler/innen dafür meist Fragewörter wie was?, wie bitte?, h?, mh? verwendet (cf. Marti 2001: 56). 
Beim "Erfragen von Zusatzinformationen" geht Marti davon aus, dass sich der/die HörerIn vor dem Nachfragen bereits eine Hypothese gebildet hat, jedoch auf weitere Informationen angewiesen ist. In dem von ihm untersuchten Korpus sind die am häufigsten gebrauchten sprachlichen Mittel Fragen wie was?, wo? wer?, warum?, jetzt? u. a. m. (cf. Marti 2001: 60).

Der Punkt "Wiederholung eines zentralen Elements" deckt sich mit der bei den Missverständnissen angeführten Strategie der Wiederholung einzelner Elemente (cf. Abschnitt 3.2).

Die impliziten Anzeiger unterteilt Marti wiederum in zwei Gruppen von Handlungsmöglichkeiten: Ignorieren und Abbrechen. Im Korpus identifiziert Marti verschiedene Symptome, die dem/der Außenstehenden helfen, implizite Anzeiger aufzudecken:

- $\quad$ "Eine Anweisung wird nicht ausgeführt oder eine Frage wird nicht als solche erkannt und beantwortet.

- $\quad$ An eine 'übergangsrelevanten Stelle' [...] wird das Rederecht nicht wahrgenommen.

- $\quad$ Der vorhergehende Redebeitrag wird nicht sinnvoll fortgesetzt.

- $\quad$ Ein unklarer oder widersprüchlicher vorhergehender Redebeitrag wird nicht korrigiert.

- $\quad$ [In einem späteren Schritt korrigiert der Sprecher den Beitrag der Hörerin und markiert ihn als Missverstehen.]" (Marti 2001: 64)

Zur Gruppe der ignorierenden impliziten Anzeiger zählt Marti "Zustimmung trotz teilweisem Nichtverstehen" und "Ignorieren und 'normal' weiterfahren - Missverständnis in Kauf nehmen". Bei beiden Reaktionen wird das Gespräch fortgeführt ohne, dass auf das Missverständnis eingegangen wird.

Die zweite Unterkategorie von impliziten Indikatoren umfasst die Reaktionen "Themawechsel", "Lachen" und "Abbruch". Genauso wenig wie ein Themawechsel notwendigerweise auf Nichtverstehen hindeuten muss, kann auch das Lachen vieldeutig sein. Wenn Lachen jedoch deplatziert wirkt, weil es z. B. auf eine direkte Frage folgt, liegt die Vermutung auf Nichtverstehen nahe.

Der Abbruch unterscheidet sich laut Marti vom Themawechsel dadurch, dass kein neues Thema begonnen wird, sondern ein altes abgeschlossen, z. B. mit dem Wort egal, wenn er/sie kein Interesse mehr (am Verstehen) bezeugt. Themawechsel, Lachen und Abbruch haben als Reaktionen gemeinsam, dass dem/der Sprechenden keine Möglichkeit zur Korrektur gegeben wird, auch wenn er/sie das Nichtverstehen erkannt hat (cf. Marti 2001: 68-72).

\section{$4 \quad$ Beispielanalysen}

Die im vorhergehenden Abschnitt analysierten Indikatoren, die Verstehen, Missverstehen und Nichtverstehen signalisieren, sollen Rückschlüsse auf verstehenshemmende Einheiten ermöglichen. Nicht immer werden Missverständnisse oder Missverstehen, auch wenn diese von einem/r der Gesprächsteilnehmer/innen wahrgenommen werden, angezeigt. "Es kommt [...] durchaus vor, dass Hörer das Nichtverstehen eines Wortsinns hinnehmen, wenn dadurch das Verständnis der Äußerungsfolge als Ganzes nicht beeinträchtigt wird. Auch aus anderen Gründen, etwa der Furcht, sich zu blamieren, oder dem Wunsch, das Thema zu wechseln oder gar das Gespräch zu beenden, werden Störungen der Sinnzuschreibung hingenommen." (Bublitz 2001: 1333). Nachdem derartige Missverständnisse für eine/n Außenstehende/n in den seltensten Fällen ersichtlich oder gar nachweisbar sein dürften, werden sie in den Untersuchungen des Visitenkorpus nicht berücksichtigt.

Anhand der oben genannten Signale sollen drei Beispiele die Identifizierung von verstehenshemmenden Einheiten im Korpus der Visitengespräche verdeutlichen: 


\section{DRICKEN 9}

Arzt

Ja ja Druck.

Pat_019 Nur Huschten und a so a bissl dricken und a sö.

Der Patient erklärt seine Beschwerten ("Nur Huschten und a so a bissl dricken und a sö"). Der Arzt reformuliert (cf. 3.1) das Problem und gibt zu Verstehen, dass er im Verb "dricken" die Bedeutung 'Druck' erkannt hat.

TUAT NET

\begin{tabular}{|llll|}
\hline $\begin{array}{l}\text { Arzt } \\
\text { Pat_009 }\end{array}$ & Wie geht s Ihnen Herr $(($ Name $)) ?$ & Jo, dr dr Fuaß tuat net. & 'Tut noch weh? \\
\hline
\end{tabular}

In diesem kurzen Ausschnitt begrüßt der Arzt seinen Patienten mit der Frage wie es ihm gehe ("Wie geht s Ihnen Herr ((Name))?"). Der Patient antwortet mit einer idiomatischen Wendung ("Jo, dr dr Fuaß tuat net") und drückt damit aus, dass sein Fuß nicht in Ordnung ist. Der Arzt reagiert mit einer Gegenfrage, in der er das Verb tun wiederholt. Mit dieser Frage will er Zusatzinformationen erfahren. Ich gehe in meiner Analyse davon aus, dass der Arzt die idiomatische Wendung - die es so in der Hochsprache nicht gibt - nur teilweise versteht, und zwar dahingehend, dass etwas nicht in Ordnung ist. Durch Wiederholung dessen, was er verstanden zu haben glaubt, versucht er über weitere Informationen das Wissensdefizit zu kompensieren. Dass der Arzt die Aussage des Patienten nicht richtig verstanden hat, ergibt sich aus der Verwendung des Wortes noch ("Tut noch weh?"), das an dieser Stelle deplatziert ist: Der Patient kann bezüglich seines Fußes noch nicht bei ihm gewesen sein, da aus dem weiteren Gesprächsverlauf ersichtlich ist, dass das Problem erst am vergangenen Wochenende aufgetaucht ist. Wäre der Patient einen oder zwei Tage zuvor gekommen, hätte sich der Arzt daran erinnert bzw. wäre das ebenfalls aus dem weiteren Gesprächsverlauf ersichtlich.

\section{IBERHEB}

\begin{tabular}{|llll|}
\hline $\begin{array}{l}\text { Arzt } \\
\text { Pat_009 }\end{array}$ & Wie geht s Ihnen Herr ((Name) $) ?$ & Jo, dr dr Fuaß tuat net. & 'Tut noch weh? \\
\hline
\end{tabular}

\begin{tabular}{|llll|}
\hline Arzt & $\bullet$ mh? & & Seit wann \\
Pat_009 & & Jo, der Fuaß do $\bullet$ hon i mi iberheb odr a Verkialung odr... & \\
\hline
\end{tabular}

\begin{tabular}{|llll|}
\hline $\begin{array}{l}\text { Arzt } \\
\text { Pat_009 }\end{array}$ & ham wir das Problem jetzt? & Ham Sie irgendwas \\
\hline
\end{tabular}

Arzt gemacht am Sonntag oder am Samstag? Können Sie sich erinnern?

Pat_009 Jaa $\bullet$ sein tuats aam Freitag hon i di Tonscheiben gsetzt und (do) i sie

\begin{tabular}{|llc|}
\hline Arzt & & Aha ham Sie s \\
Pat_009 no amol auheb hon i an Riss krieg odr a Verkialung... & \\
\hline
\end{tabular}

\begin{tabular}{|lll|}
\hline Arzt & gemerkt, dass Sie ((unverständlich))... & \\
Pat_009 & & Obr obr in Schmerz hon i/ isch erscht in \\
\hline
\end{tabular}

Pat_009 Sonntag obends kommen.

\footnotetext{
${ }^{9}$ Die Gespräche wurden nach HIAT in EXMARaLDA transkribiert.
} 
Der Patient erklärt, warum er Probleme mit seinem Fuß hat ("hon i mi iberheb odr a Verkialung"). Er verwendet das Wort iberheb und meint damit, dass er zu schwer getragen hat. Der Arzt ignoriert vorerst das Gesagte, reagiert mit einer Gegenfrage ("Seit wann ham wir das Problem jetzt?") und fragt gleich anschließend, wie es zu den Schmerzen gekommen sei ("Ham Sie irgendwas gemacht am Sonntag oder am Samstag? Können Sie sich erinnern?"). Diese Information hat der Patient allerdings eingangs bereits gegeben ("hon i mi iberheb"). Im Folgenden beschreibt der Patient dann ausführlich, wie es zum Problem gekommen ist. Aus den Daten lässt sich herauslesen, dass der Arzt das Verb "hon mi iberheb" nicht verstanden hat und daraufhin mit einem Themawechsel (cf. 3.3) eingeleitet durch eine Gegenfrage das Gesagte ignoriert.

\section{$5 \quad$ Fazit}

Die vorgeschlagene Untersuchung zur Identifizierung von verstehenshemmenden sprachlichen Einheiten anhand von Signalen in der Reaktion des/r Hörenden erfasst schwer verständliche Einheiten sicher nicht vollständig und kann nur komplementär als eine von mehreren Methoden auf der Suche nach derselben angewendet werden. Verschiedene Hintergrundinformationen zu den Gesprächen, die über Schwierigkeiten beim Verstehen Aufschluss geben können, wurden in diesem Beitrag gar nicht angesprochen. Erkenntnisse von Forschungsansätzen und -schwerpunkten sowie Methoden wie der Dialoganalyse, der interkulturellen Kommunikation, Hörverstehen in einer Fremdsprache, Semikommunikation, Dialektalitätsmessung, Eurocom u. a. m., könnten sicherlich zusätzliche Erkenntnisse bringen, die für die Analyse von schwer zu verstehenden Einheiten eine Rolle spielen.

Allein die Tatsache, dass - wie erwähnt - unter verschiedenen Umständen Missverständnisse und Nichtverstehen nicht manifest gemacht werden, obwohl sie den Gesprächsteilnehmer/innen bewusst sind, macht deutlich, dass die in Ansätzen dargestellte Systematisierung von Missverständnissen viel komplexer ist als sie hier thematisiert wurde und auf einen ersten Blick erscheinen mag (cf. Hinnenkamp 1998).

\section{$6 \quad$ Literaturangaben}

Bublitz, Wolfram (2001): "Formen der Verständnissicherung in Gesprächen. In: Brinker, Klaus/Antos, Gerd/Heinemann, Wolfgang/Sager, Sven F. (eds.): Text- und Gesprächslinguistik. Ein internationales Handbuch zeitgenössischer Forschung. 2. Halbband. Berlin/New York: 1330-1340.

Deppermann, Arnulf (im Druck): "Verstehen im Gespräch. Erscheint in: Eichinger, Ludwig M./Kämper, Heidrun (eds.) (im Druck): Sprache - Kognition - Kultur. Jahresbuch des Instituts für Deutsche Sprache 2007. Tübingen.

Falkner, Wolfgang (2007): "Missverstehenshermeneutik". In: Hermanns, Fritz/Holly, Werner (2007) (eds.): Linguistische Hermeneutik. Theorie und Praxis des Verstehens und Interpretierens. Tübingen: 175-200. (= Reihe Germanistische Linguistik 272).

Hinnenkamp, Volker (1998): Missverständnisse in Gesprächen. Eine empirische Untersuchung im Rahmen der interpretativen Soziolinguistik. Wiesbaden.

Linz, Erika (2007): "Sich sprechend verstehen. Zur Nachträglichkeit des Äußerungssinns in der Rede". In: Hermanns, Fritz/Holly, Werner (2007) (eds.): Linguistische Hermeneutik. Theorie und Praxis des Verstehens und Interpretierens. Tübingen: 43-58. (= Reihe Germanistische Linguistik 272).

Marti, Jakob (2001): Verstehensschwierigkeiten bei Nicht-MuttersprachlerInnen. Eine empirische Studie anhand von Aufnahmen bei Asylsuchenden in Bern. Lizentiatsarbeit, Universität Bern. http://home.datacomm.ch/jakob.marti, Stand 13.12.2007. 
Rath, Rainer (2001): "Gesprächsschritt und Höreraktivitäten". In: Brinker, Klaus et al. (eds.): Text- und Gesprächslinguistik. Ein internationales Handbuch zeitgenössischer Forschung. 2. Halbband. Berlin/New York. 1213-1226.

Rehbein, Jochen (1985): Interkulturelle Kommunikation. Tübingen.

Rost-Roth, Martina (1994): "Verständigungsprobleme in der interkulturellen Kommunikation. Ein Forschungsüberblick zu Analysen und Diagnosen in empirischen Untersuchungen". In: LiLi. Zeitschrift für Literaturwissenschaft und Linguistik 24/93:9-45.

Rost-Roth, Martina (2002): "Kommunikative Störungen in Beratungsgesprächen. Problempotentionale in inter- und intrakulturellen Gesprächskontexten". In: Fiehler, Reinhard (ed.): Verständigungsprobleme und gestörte Kommunikation. Radolfzell: 216244.

Schäflein-Armbruster, Robert (1994): "Dialoganalyse und Verständlichkeit". In: Fritz, Gerd/Hundsnurscher, Franz (eds.): Handbuch der Dialoganalyse. Tübingen: 493-518.

Selting, Margret (1987): Verständigungsprobleme. Eine empirische Analyse am Beispiel der Bürger-Verwaltungs-Kommunikation. Tübingen. 